\title{
Semiautomated Evaluation of the Primary Motor Cortex in Patients with Amyotrophic Lateral Sclerosis at 3T
}

\author{
(D) G. Donatelli, (D) A. Retico, (DE. Caldarazzo lenco, (D). Cecchi, (D). Costagli, (DD. Frosini, (D). Biagi, (D). Tosetti, (D) G. Siciliano, and \\ (D) M. Cosottini

\begin{abstract}
BACKGROUND AND PURPOSE: Amyotrophic lateral sclerosis is a neurodegenerative disease involving the upper and lower motor neurons. In amyotrophic lateral sclerosis, pathologic changes in the primary motor cortex include Betz cell depletion and the presence of reactive iron-loaded microglia, detectable on 7T MR images as atrophy and T2*-hypointensity. Our purposes were the following: 1) to investigate the signal hypointensity-to-thickness ratio of the primary motor cortex as a radiologic marker of upper motor neuron involvement in amyotrophic lateral sclerosis with a semiautomated method at 3T, 2) to compare 3T and 7T results, and 3) to evaluate whether semiautomated measurement outperforms visual image assessment.
\end{abstract}

MATERIALS AND METHODS: We investigated 27 patients and 13 healthy subjects at 3T, and 19 patients and 18 healthy subjects at 7T, performing a high-resolution 3D multiecho $\mathrm{T}^{*}$-weighted sequence targeting the primary motor cortex. The signal hypointensity-tothickness ratio of the primary motor cortex was calculated with a semiautomated method depicting signal intensity profiles of the cortex. Images were also visually classified as "pathologic" or "nonpathologic" based on the primary motor cortex signal intensity and thickness.

RESULTS: The signal hypointensity-to-thickness ratio of the primary motor cortex was greater in patients than in controls ( $P<.001)$, and it correlated with upper motor neuron impairment in patients $(\rho=0.57, P<.001)$. The diagnostic accuracy of the signal hypointensityto-thickness ratio was high at $3 \mathrm{~T}$ (area under the curve $=0.89$ ) and even higher at 7T (area under the curve $=0.94$ ). The sensitivity of the semiautomated method (0.81) outperformed the sensitivity of the visual assessment $(0.56-0.63)$ at $3 T$.

CONCLUSIONS: The signal hypointensity-to-thickness ratio of the primary motor cortex calculated with a semiautomated method is suggested as a radiologic marker of upper motor neuron burden in patients with amyotrophic lateral sclerosis. This semiautomated method may be useful for improving the subjective radiologic evaluation of upper motor neuron pathology in patients suspected of having amyotrophic lateral sclerosis.

ABBREVIATIONS: ALS = amyotrophic lateral sclerosis; $\mathrm{HS}=$ healthy subjects; $\mathrm{Ml}=$ primary motor cortex; $\mathrm{ROC}=$ receiver operating characteristic; $\mathrm{SH} / \mathrm{Thk}=$ signal hypointensity-to-thickness ratio; UMN = upper motor neuron

A myotrophic lateral sclerosis (ALS) is a progressive and clinically heterogeneous neurodegenerative disease involving both upper and lower motor neurons, ${ }^{1,2}$ having different prognoses $^{3}$ and, perhaps, different responses to possible therapies, even

Received May 3, 2017; accepted after revision August 13.

From the Department of Translational Research and New Technologies in Medicine and Surgery (G.D., P.C., M. Cosottini) and Neurology Unit, Department of Clinical and Experimental Medicine (E.C.I., D.F., G.S.), University of Pisa, Pisa, Italy; National Institute for Nuclear Physics (A.R.), Pisa Division, Pisa, Italy; Imago7 Research Foundation (M. Costagli, L.B., M.T.), Pisa, Italy; and Laboratory of Medical Physics and Biotechnologies for Magnetic Resonance (M. Costagli, L.B., M.T.), Istituto di Ricovero e Cura a Carattere Scientifico Stella Maris, Pisa, Italy.

This work is part of the experimental protocol "Clinical Impact of Ultra-High-Field MRI in Neurodegenerative Diseases Diagnosis" (RF RF-2009-1546281) approved and funded by the Italian Ministry of Health and cofunded by the Health Service of Tuscany.

Paper previously presented at: European Congress of Radiology, March 1-5, 2017; Vienna, Austria. in the experimental scenario. Different from the lower motor neuron impairment that can be carefully investigated with electrophysiologic tests, ${ }^{4}$ the evaluation of upper motor neuron (UMN) burden is mainly clinical ${ }^{1,5}$ and is partially confounded by signs related to lower motor neuron degeneration. ${ }^{4}$ Moreover, at the time of the diagnosis, the UMN impairment can range widely from faint to severe, and the variability in signs and symptoms at disease onset ${ }^{3}$ makes early diagnosis and correct phenotypic characterization of the disease difficult.

In ALS, the main pathologic changes in the primary motor

Please address correspondence to Graziella Donatelli, MD, Neuroradiology Unit, Via Paradisa 2, 56124, Pisa, Italy; e-mail: graziella_donatelli@hotmail.com

- Indicates open access to non-subscribers at www.ajnr.org

三 Indicates article with supplemental on-line tables.

Indicates article with supplemental on-line photos.

http://dx.doi.org/10.3174/ajnr.A5423 
cortex (M1) include the loss of Betz cells in the layer $\mathrm{V}^{6}$ and the presence of reactive iron-loaded microglia, ${ }^{6,7}$ visible on MR images as cortical atrophy ${ }^{8-10}$ and $\mathrm{T} 2{ }^{*}$ hypointensity, ${ }^{7}$ respectively. In recent years, several conventional and nonconventional MR imaging techniques have been used to look for a biomarker of UMN impairment at both cortical and subcortical levels with variable results, and quantitative measurements of cortical atrophy were performed at a group level. ${ }^{11}$ Despite such effort, a definite and reliable marker of UMN degeneration is not yet available. As a result, while MR imaging of the brain is currently used to exclude mimic pathology and the detection of the T2 hypointensity of M1 can support suspicion of ALS, the specific search for this abnormality is not recommended for ALS diagnosis. ${ }^{12}$

The first attempt to move toward the radiologic diagnosis at the single-subject level was recently performed with an ultrahigh-field MR imaging system (7T). ${ }^{13}$ Taking advantage of the very high sensitivity of ultra-high-field strength to the magnetic susceptibility of microglial ferritin, the authors localized pathologic cortical thinning and $\mathrm{T} 2{ }^{\star}$ hypointensity in the deep layers of M1, and they were shown to significantly correlate with the clinical UMN burden. In light of these results, the $\mathrm{T} 2{ }^{\star}$ hyopintensity of $\mathrm{M} 1$ was suggested as a possible marker of neuroinflammation and UMN impairment in patients with ALS rather than a marker of the disease. Unfortunately, the $\mathrm{T} 2^{*}$ sensitivity to microglial ferritin depends on the magnetic field strength, and M1 assessment in patients with ALS can be a challenge in clinical practice using MR imaging systems up to $1.5 \mathrm{~T}$ also in patients with a severe UMN impairment. On the contrary, 3T scanners may change the radiologic approach to patients with pyramidal symptoms and signs in motor neuron diseases. Therefore, our main aim was to investigate the signal hypointensity-to-thickness ratio ( $\mathrm{SH} / \mathrm{Thk})$ of the deep layers of the M1 as a radiologic marker of UMN burden in patients with ALS with a semiautomated method at 3T. Secondary aims were to evaluate whether the results obtained with a clinical scanner (3T) were comparable with those achieved with a research scanner (7T) and whether the semiautomated measurements improved the sensitivity of the visual radiologic assessment.

\section{MATERIALS AND METHODS \\ Patients with ALS and Healthy Subjects}

Twenty-seven patients with ALS (18 men and 9 women; mean age, $58 \pm 11$ years) and 13 healthy subjects (HS; 6 men and 7 women; mean age, $56 \pm 15$ years) underwent brain MR imaging with a $3 \mathrm{~T}$ system.

Nineteen patients with ALS (14 men and 5 women; mean age, $63 \pm 10$ years), different from the patients with ALS investigated at 3T, and $18 \mathrm{HS}$ ( 9 men and 9 women; mean age, $56 \pm 13$ years) underwent brain MR imaging with a $7 \mathrm{~T}$ system.

Five of the above-mentioned HS underwent both examinations to compare the performance of the $3 \mathrm{~T}$ and $7 \mathrm{~T}$ systems in the assessment of signal intensity and thickness of the deep layers of M1.

All patients had a diagnosis of definite ALS according to the revised El Escorial criteria ${ }^{1}$ and were consecutively enrolled by the neurology unit of the University Hospital of Pisa. They were clinically evaluated by an experienced neurologist on the day of en- rollment, and the UMN impairment was quantified for each limb using a clinical composite semiquantitative arbitrary score of UMN burden (UMN score), according to that previously used by Cosottini et al. ${ }^{13}$ For each patient, the total UMN score (range, $0-33$ ) and the UMN score of each limb (range, 0-8) were recorded; then, the average UMN score was calculated as the mean of UMN scores of both arms and legs. Clinical and demographic data of patients are reported in On-line Tables 1-3. Exclusion criteria for enrollment were the presence of neurologic comorbidities. HS were enrolled from among relatives and spouses of patients with ALS and radiology department staff; none had any history of neurologic or psychiatric diseases.

All patients and controls gave their written informed consent for the enrollment. This study was performed as part of the experimental protocol called "Clinical Impact of Ultra-High-Field MRI in Neurodegenerative Diseases Diagnosis,” RF-2009-1546281, approved by Italian Ministry of Health and by the local ethics committee. The project was founded by the Italian Ministry of Health and cofunded by the Health Service of Tuscany.

\section{MR Imaging Acquisition}

The MR imaging protocol at both $3 \mathrm{~T}$ and $7 \mathrm{~T}$ included a 3D multiecho $\mathrm{T} 2{ }^{*}$-weighted sequence prescribed axially and covering the brain from the vertex to the splenium of the corpus callosum.

MR imaging examinations at $3 \mathrm{~T}$ were performed with a Discovery MR 750 (GE Healthcare, Milwaukee, Wisconsin) scanner equipped with an 8-channel head coil. Acquisition parameters of the $3 \mathrm{D}$ multiecho $\mathrm{T} 2^{*}$-weighted sequence were the following: $\mathrm{TR}=68.3 \mathrm{~ms}$; TEs $=13,18.6,24.3,29.9,35.5,41.2,46.8,52.4$, $58.1,63.7 \mathrm{~ms}$; flip angle $=15^{\circ}$; NEX $=0.70$; acquisition matrix $=$ $448 \times 384 ; \mathrm{FOV}=20 \times 20 \mathrm{~cm}$; spatial resolution of reconstructed images $=0.39 \times 0.39 \times 1 \mathrm{~mm}^{3}$; scan duration $=4$ minutes 22 seconds.

MR imaging examinations at 7T were performed with a Discovery MR 950 scanner (GE Healthcare) equipped with a $2 \mathrm{CH}$ TX/32CH-RX head coil (Nova Medical, Wilmington, Massachusetts). Technical parameters of the $3 \mathrm{D}$ multiecho $\mathrm{T}^{\star}{ }^{\star}$-weighted sequence were the following: $\mathrm{TR}=54.1 \mathrm{~ms}$; TEs $=5.6,12,18.3$, $24.7,31.1,37.5$, $43.9 \mathrm{~ms}$; flip angle $=15^{\circ}$; $\mathrm{NEX}=0.70$; acquisition matrix $=448 \times 448 ; \mathrm{FOV}=22.4 \times 22.4 \mathrm{~cm}$; spatial resolution of reconstructed images $=0.5 \times 0.5 \times 1 \mathrm{~mm}^{3}$; scan duration $=6$ minutes 59 seconds.

\section{Semiautomated Image Assessment}

In each hemisphere of all subjects, the thickness and signal intensity of the regions of M1 corresponding to Penfield areas of the upper $^{14}$ and lower ${ }^{15}$ limbs were assessed with an in-house-developed, semiautomated tool for image processing. Given the wide cortical extension of the upper and lower limb motor areas, to increase the reliability of the ROI position among subjects, we selected 2 smaller M1 subregions for the ROI positioning; thus, ROIs of the upper limbs were positioned in the hand knob, whereas ROIs of the lower limbs were positioned in the most cranial and lateral part of the paracentral lobule. In each set of images, a neuroradiologist blinded to the clinical diagnosis identified the sections that best represented each M1 target region and an additional section including the splenium of the corpus callo- 
sum, which served to obtain 1 region of reference for the M1 signal intensity measures. ${ }^{16}$ The interactive image-processing tools were run according to the steps described in detail in On-line Fig 1. The observer is prompted to draw a polygonal ROI (not $<5$ $\mathrm{mm}^{2}$ ) in the splenium of the corpus callosum, whose average intensity was retained to normalize the intensity values of the cortex ROIs. For each M1 target region, the observer manually draws the profile of the interface between the M1 and the neighboring subarachnoid space, which is interpolated by the software with a spline function to make it smoother. The directions normal to the spline are computed, and the trend of the signal intensity (y-axis) is reported as the function of the distance along the normal direction to the cortex ( $\mathrm{x}$-axis) in physical units (millimeter). The signal intensity profiles are then averaged and the absolute value is considered. A baseline intensity value corresponding to the average signal intensity of the subcortical white matter, computed in a region chosen by the observer, is then subtracted. The hypointensity profile is then fitted with a double sigmoid function $F$ that can be expressed as the difference between 2 sigmoid function as follows: $F\left(x ; a, b_{1}, b_{2}, c_{1}, c_{2}\right)=a\left[f_{1}\left(x ; b_{1}, c_{1}\right)-f_{2}\left(x ; b_{2}, c_{2}\right)\right]$, where $f(x ; b, c)=1 /\left\{1+e^{\wedge}[-b(x-c)]\right\}$. The thickness and the height of the final intensity profile, which was normalized with respect to the signal intensity of the splenium of the corpus callosum, are recorded as measures of the mean thickness and signal hypointensity of M1 deep layers, respectively. In particular, the depth of the curve and the width at $25 \%$ of the height are taken as measures of signal hypointensity and thickness of the deep layers of the M1, respectively.

The semiautomated tool for image processing was implemented in Matlab (MathWorks, Natick, Massachusetts) and runs on different operating systems (Windows, MacOS, Linux). It receives input MR images in the NIfTI or Analyze (AnalyzeDirect, Overland Park, Kansas) file format and returns the measures of the cortex hypointensity and thickness as well as several graphic representations of intermediate steps of the interactive image processing.

For each target region of every subject, the SH/Thk was calculated to put together and maximize the contribution of both parameters in the morphometric assessment of the cortex. In the comparison between patients and HS and in the computation of the receiver operating characteristic (ROC) curve, we included in the analysis all data of all HS and, for each patient, only the greatest SH/Thk value recorded between the 2 hemispheres. The choice of selecting only 1 measure for each patient was based on the possible asymmetry in the pathologic involvement of the M1. ${ }^{17-19}$ On the contrary, all measures of all patients were used in assessing the correlation between the SH/Thk of the M1 subregions and the $\mathrm{UMN}$ scores of the corresponding limbs.

\section{Visual Image Assessment}

All images were randomly evaluated by 2 neuroradiologists ( $M$. Cosottini and G.D., with 26 and 7 years of experience, respectively) blinded to the clinical diagnosis. The observers were asked to visually evaluate each series of images on the basis of the signal intensity and thickness of the deep layers of M1 compared with those of neighboring cortices. Images were labeled as "nonpathologic" if M1 deep layers were judged to be similar to those of other

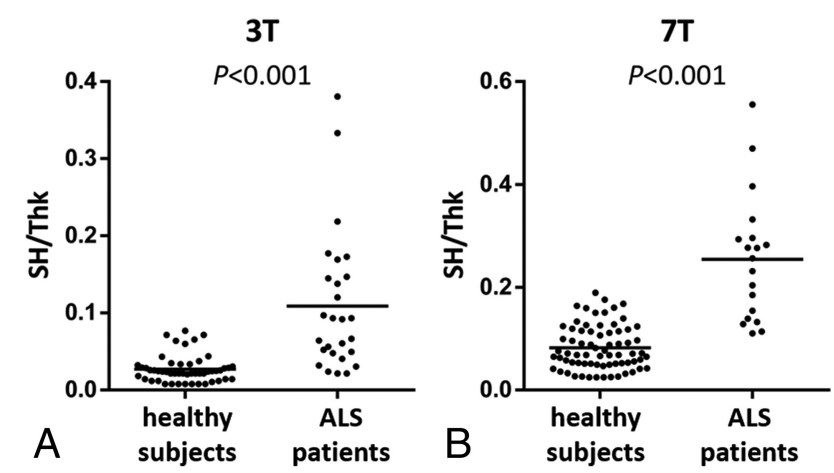

FIG 1. Boxplot of the SH/Thk measured in the deep layers of the primary motor cortex in healthy subjects and patients with ALS at 3T $(A)$ and $7 T(B)$. At both magnetic fields, the ratio is significantly greater in patients than in subjects $(P<.001)$.

cortices or "pathologic" if they were markedly more hypointense and thin. ${ }^{13}$ After 1 month from the first reading, the same neuroradiologists were asked to again assess the whole set of images.

\section{Statistical Analysis}

Quantitative and semiquantitative data were analyzed using nonparametric statistical tests with the significance level set to .05. In more detail, 3T and 7T data concerning HS who underwent both MR imaging examinations were compared using the Wilcoxon test. The SH/Thk comparison between patients with ALS and HS was performed using the Friedman test, whereas all other intergroup comparisons were investigated using the Mann-Whitney $U$ test. Relationships between variables were investigated with the Spearman rank test, and ROC analysis was used as a binary classifier system to evaluate the performance of the SH/Thk in distinguishing patients from HS.

Using the clinical diagnosis as the criterion standard, we calculated the sensitivity, specificity, positive predictive value, negative predictive value, and diagnostic accuracy of the visual assessment of images for both reading sessions of both observers (for each reader, data shown in the "Results" section refer to the reading session with the best diagnostic accuracy). The Cohen $\kappa$ statistic was used to calculate intra- and interrater reliability.

\section{RESULTS}

\section{Epidemiologic and Clinical Data Analysis}

The age of patients was not significantly different from that of HS at both 7T and 3T ( $P=.12$ and $P=.64$, respectively). Total UMN scores and mean UMN scores did not significantly differ between patients investigated at $3 \mathrm{~T}$ and patients assessed at $7 \mathrm{~T}(P=.87$ and $P=.86$, respectively).

\section{Semiautomated Image Assessment}

\section{$\mathrm{SH} /$ Thk of the Primary Motor Cortex.}

3T. SH/Thk was significantly higher in patients than in HS (mean, $0.11 \pm 0.09 ; 95 \% \mathrm{CI}, 0.07-0.14$ for patients; and mean, $0.03 \pm 0.02 ; 95 \%$ CI, 0.02-0.03 for HS; $P<.001$ ) (Fig $1 A$ ).

7T. SH/Thk was significantly higher in patients than in HS (mean, $0.25 \pm 0.12 ; 95 \% \mathrm{CI}, 0.20-0.31$ for patients; and mean, $0.08 \pm 0.04$; 95\% CI, 0.07-0.09 for HS; $P<.001$ ) (Fig 1B). 
In the subgroup of HS who underwent MR imaging at both 3T and 7T, the SH/Thk of the deep layers of the M1 was significantly $(P<.001)$ higher at $7 \mathrm{~T}$ than at 3T.

\section{Correlation of the SH/Thk with UMN Score.}

3T. In patients with ALS, the SH/Thk of the deep layers of M1 significantly correlated with the UMN score of the corresponding limbs ( $\rho=0.57, P<.001 ; 95 \%$ CI, 0.42-0.69) (Fig 2A).

7T. In patients with ALS, the SH/Thk measured in the M1 deep layers showed a significant correlation with the UMN score of the corresponding limbs $(\rho=0.57, P<.001$; 95\% CI, $0.39-$ 0.71) (Fig 2B).

The measures of signal intensity and thickness of M1 subregions of HS overlapped those recorded in cortical subregions of patients corresponding to limbs with UMN scores of up to 3. On the contrary, the dataset of HS was more clearly distinguishable from that concerning cortical subregions of patients correspond-
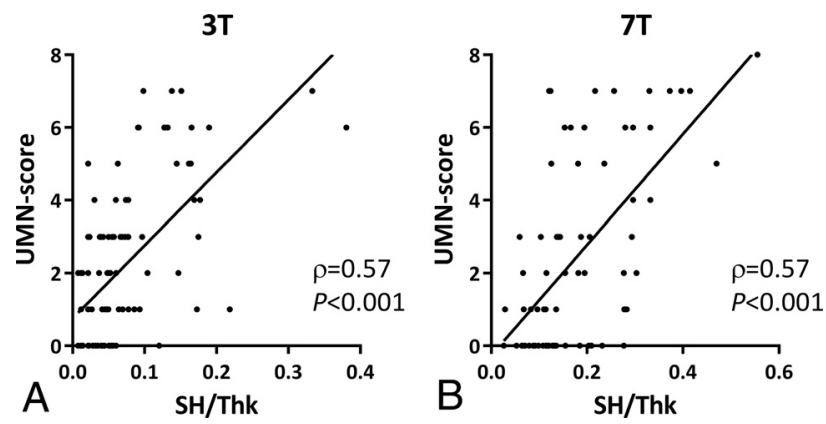

FIG 2. Correlation between the SH/Thk measured in the deep layers of the primary motor cortex of patients with ALS (hand knob and paracentral lobule of both hemispheres) and the UMN scores of the corresponding limbs. Correlations are moderate and significant at both 3T and 7T $(\rho=0.57, P<.001)$.
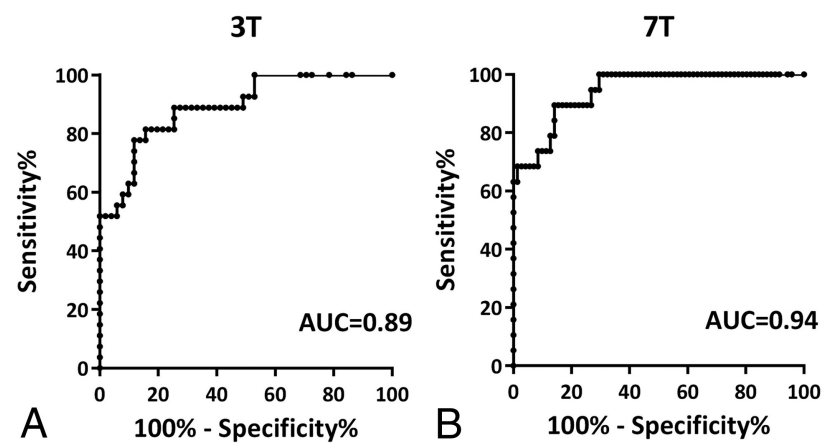

FIG 3. ROC curves elaborated with $3 T(A)$ and $7 T(B)$ data pool. The curves show, respectively, a good and an excellent accuracy to discriminate pathologic and nonpathologic values of SH/Thk. ing to limbs with UMN scores of $>3$. This observation was seen with both $3 \mathrm{~T}$ and $7 \mathrm{~T}$ data (On-line Figs 2 and 3).

\section{Diagnostic Performance of SH/Thk.}

3T. ROC analysis of 3T data showed a good accuracy (area under the curve $=0.89)$ in differentiating pathologic from nonpathologic data, with a sensitivity of 0.81 and a specificity of 0.84 (Fig $3 A$ ).

7T. ROC analysis of 7T data showed an excellent accuracy (area under the curve $=0.94)$ as binary classifiers of data as pathologic or nonpathologic, with a sensitivity of 0.89 and a specificity of 0.86 (Fig 3B).

\section{Visual Image Assessment}

3T. Sensitivity, specificity, and diagnostic accuracy were, respectively, $0.56,0.92$, and 0.68 for the first reader and $0.63,0.85$, and 0.70 for the second reader (Table). The intrarater agreement was good for the first reader and very good for the second reader $(0.74$ and 0.85 , respectively), and the interrater agreement was good for both reading sessions $(0.80$ and 0.70$)$. The mean values of the total UMN scores of patients correctly diagnosed and patients misdiagnosed were, respectively, 11 and 5 for the first reader and 12 and 3 for the second reader.

7T. Sensitivity, specificity, and diagnostic accuracy were, respectively, $0.68,0.89$, and 0.78 for the first reader and $0.63,1.00$, and 0.81 for the second reader (Table). The intrarater agreement was very good for both readers $(0.83$ and 0.81$)$, and the interrater agreement was good for both reading sessions (0.65 and 0.77 ). The mean values of the total UMN scores of patients correctly diagnosed and patients misdiagnosed were, respectively, 13 and 2 for the first reader and 13 and 4 for the second reader.

\section{DISCUSSION}

\section{SH/Thk Changes in Patients with ALS and Correlation with Clinical UMN Impairment}

The magnetic susceptibility of the deep layers of the M1, revealed by $3 \mathrm{D}$ multiecho $\mathrm{T} 2{ }^{\star}$-weighted images and related to cortical content of nonheme iron, ${ }^{7,16,20}$ can be used to assess both their signal intensity and thickness. The distinction between the superficial and deep layers of the M1 is also often detectable on 3T images of patients and HS (On-line Fig 1 and Fig 4) and depends on the amount of myelinated fibers, deep layers being more myelinated than superficial ones. The gray-white matter junction in the M1 is sometimes hardly distinguishable in HS and in patients with mild UMN impairment because of the heavily myelinated deep layers of M1. ${ }^{13}$ On the contrary, in patients with ALS with moderate-tosevere UMN impairment, the deep layers of the M1 appear mark-

Results of visual assessment of $3 \mathrm{~T}$ and $7 \mathrm{~T}$ images

\begin{tabular}{|c|c|c|c|c|c|c|c|c|}
\hline & \multicolumn{4}{|c|}{$3 T$} & \multicolumn{4}{|c|}{$7 \mathrm{~T}$} \\
\hline & \multicolumn{2}{|c|}{ First Reader } & \multicolumn{2}{|c|}{ Second Reader } & \multicolumn{2}{|c|}{ First Reader } & \multicolumn{2}{|c|}{ Second Reader } \\
\hline & $\begin{array}{c}\text { First } \\
\text { Reading }\end{array}$ & $\begin{array}{l}\text { Second } \\
\text { Reading }\end{array}$ & $\begin{array}{c}\text { First } \\
\text { Reading }\end{array}$ & $\begin{array}{l}\text { Second } \\
\text { Reading }\end{array}$ & $\begin{array}{c}\text { First } \\
\text { Reading }\end{array}$ & $\begin{array}{l}\text { Second } \\
\text { Reading }\end{array}$ & $\begin{array}{c}\text { First } \\
\text { Reading }\end{array}$ & $\begin{array}{l}\text { Second } \\
\text { Reading }\end{array}$ \\
\hline Sensitivity & 0.52 & 0.56 & 0.63 & 0.59 & 0.63 & 0.68 & 0.63 & 0.58 \\
\hline Specificity & 0.92 & 0.92 & 0.85 & 0.85 & 0.89 & 0.89 & 1.00 & 1.00 \\
\hline Positive predictive value & 0.93 & 0.94 & 0.89 & 0.89 & 0.86 & 0.87 & 1.00 & 1.00 \\
\hline Negative predictive value & 0.48 & 0.50 & 0.52 & 0.50 & 0.70 & 0.73 & 0.72 & 0.69 \\
\hline Diagnostic accuracy & 0.65 & 0.68 & 0.70 & 0.68 & 0.76 & 0.78 & 0.81 & 0.78 \\
\hline
\end{tabular}




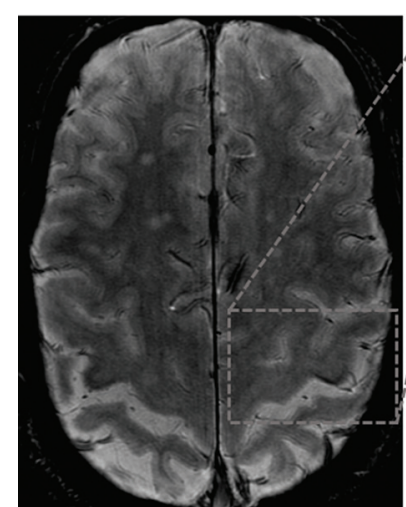

A

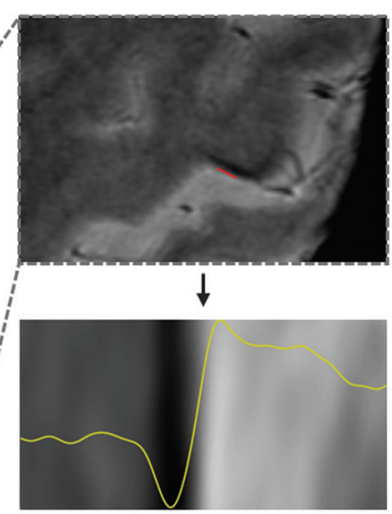

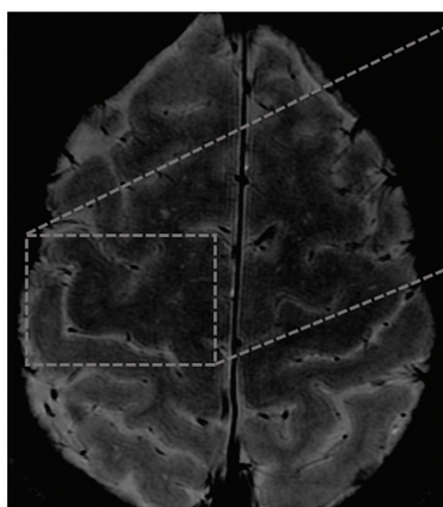

B

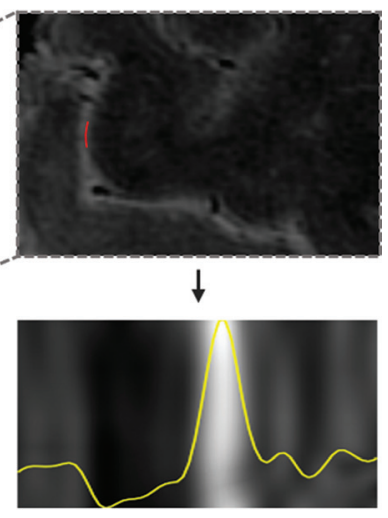

FIG 4. The signal profile of the MI containing the intensity and thickness information is shown for the left knob of a patient with ALS (A) and the right knob of a healthy subject $(B)$, where the deep layers of the cortex are clearly visible.

edly more hypointense than the underlying white matter (On-line Fig 1 and Fig 4).

When we used such cortical features in the semiautomated analysis, the SH/Thk was significantly higher in patients than in HS. This finding confirmed its usefulness in the correct assessment of M1 morphology at the group level. However, as shown in Fig 1, SH/Thk values were more scattered in patients than in HS, and there was a partial overlap between the 2 groups. Such distribution of patient data was most likely due to the nonuniform UMN burden among patients. In fact, as shown in On-line Fig 3, a greater UMN score corresponded to a greater ability to distinguish patients from HS, mainly for UMN scores of $>3$. From a clinical point of view, in the group of patients with ALS, there was a significant positive correlation between the SH/Thk of the deep layers of M1 and the UMN score of the corresponding limbs: the greater the SH/Thk, the greater the clinical limb impairment. In other words, the cortical hypointensity ranged from being very pronounced to being seemingly indistinguishable from that of an unaffected cortex in patients with severe or light UMN impairment, respectively.

The hypothesis of a direct link between the location of cortical atrophy within the motor homunculus and clinical signs of UMN impairment was proposed $>20$ years ago on the basis of pathologic studies, ${ }^{17}$ but until now, only a few MR imaging studies investigated such a correlation using scores of functional disability ${ }^{9,10,21}$ or UMN impairment. ${ }^{13}$ Our results agree with previous findings at ultra-high-field MR imaging ${ }^{13}$ and confirm the link between the degree of focal cortical atrophy and hypointensity in the motor homunculus and the degree of signs of UMN degeneration in the corresponding limbs. Such results explain why neuroradiologists can correctly diagnose patients with ALS with moderate-to-severe UMN impairment, whereas patients with low or very low UMN burden are misdiagnosed. Moreover, the pattern of $\mathrm{T}^{\star}$ hypointensity can be different among patients. In fact, according to the UMN burden, the extension of M1 signal hypointensity ranges from being localized to a small region of the M1 to bilaterally involving its full length, from the interhemispheric fissure to the lateral sulcus.

The marked $\mathrm{T} 2^{\star}$ hypointensity of the deep layers of M1 in some patients with ALS compared with HS was demonstrated to be the expression of the greater magnetic susceptibility related to the abundant intracortical deposition of iron in the form of microglial ferritin. ${ }^{7} 16$ With the magnetic susceptibility having a positive and strict dependence on the magnetic field strength, $\mathrm{T} 2{ }^{*}$ sensitivity to paramagnetic substances is lower at $3 \mathrm{~T}$ than at $7 \mathrm{~T}$, as demonstrated in the subgroup of HS who underwent MR imaging examinations at both magnetic fields, thus reducing the ability to detect small collections of intracortical ferritin in patients with ALS with moderate-to-low UMN impairment. Such findings explain why the sensitivity, negative predictive value, and diagnostic accuracy in the visual imaging assessment were lower at $3 \mathrm{~T}$ than at 7T. In line with this result, the performance of ROC analysis was also slightly better at $7 \mathrm{~T}$ than at $3 \mathrm{~T}$. However, the accuracy in distinguishing pathologic and nonpathologic images using the semiautomated method was also high at $3 \mathrm{~T}$.

ROC data can be used in the assessment of subjects referred from neurologists with suspected motor neuron disease. In this scenario, the choice of a cutoff that improves sensitivity though affecting specificity can result in a more accurate M1 evaluation of patients. In fact, in our population, the use of the semiautomated method showed an increased sensitivity in evaluating M1 morphologic changes compared with visual imaging assessment. Therefore, besides visual image evaluation, after data collection from healthy subjects and the definition of a cutoff value, the use of the SH/Thk in clinical practice can contribute to the radiologic evaluation of images, mainly in patients with mild UMN burden, confirming morphologic changes that are only slightly visible on visual inspection. More interesting prospects are to increase the sensitivity of neuroimages in the detection of UMN pathology, allowing the identification of very small M1 changes and reducing the false-negative rate, and to estimate the UMN burden, thus supporting the clinical evaluation of patients and contributing to their phenotypical classification. To further support this hypothesis, one could confirm, in a larger sample, that the UMN score of patients correctly classified at visual assessment is higher than that of patients in whom visual and semiautomated assessments disagree in the classification of images.

One should note the following aspects of the present study. First, different from studies investigating cortical thickness, which assessed the cortex in a full-thickness fashion, ${ }^{8-10}$ here we evaluated only the deep layers of M1, known to be the location of pathologic changes. Second, contrary to cortical thickness and 
voxel-based morphometry studies, ${ }^{8-10,18,19}$ after having collected some data from HS, the presented method could be applied not only at the group level but also at the single-subject level to estimate the UMN burden in each patient with pyramidal signs and symptoms. Third, until now, MR imaging studies investigated, individually, cortical atrophy ${ }^{8-10,18,19}$ or hypointensity. ${ }^{7,22}$ To the best of our knowledge, this is the first study assessing the combination of both parameters, thus improving the radiologic evaluation of M1. Compared with the assessment of a single parameter (SH or Thk), the use of the SH/Thk gives us 3 main advantages: 1 ) to assess simultaneously 2 different radiologic features related to the cortical neurodegeneration; 2) to find a radiologic tool that correlates with clinical UMN impairment; and 3) to reduce the false-positive ratio related to the increase in $\mathrm{T} 2{ }^{\star}$ hypointensity of M1 in the elderly. Furthermore, because the SH/Thk is semiquantitative data measured in each single subject, it could be used for the phenotypic stratification of UMN involvement in longitudinal studies aiming at investigating the spread of cortical changes across time ${ }^{2}$ or the efficacy of neuronal and nonneuronal therapies.

\section{Methodologic Considerations}

$\mathrm{T}^{*}$ signal features within the cortex allow distinguishing superficial and deep layers, thus measuring only the thickness of M1 deep layers, where the atrophy seems to be localized. By contrast, sequences commonly used for cortical thickness measurements, such as inversion recovery T1-weighted sequences, provide better gray-white matter contrast but are used only for full-thickness cortical measurements and do not allow more targeted measurements.

For evaluation of cortical thinning and signal hypointensity of the deep layers of the primary motor cortex at 7T, 2D gradient recalled sequences with high in-plane resolution have previously been used. ${ }^{13}$ However, in clinical settings on high-field MR imaging systems (3T), 2D gradient recalled-echo sequences are not as efficient as 3D multiecho $2^{\star}{ }^{\star}$-weighted techniques, which were proved to be the most sensitive in the detection of the low signal intensity in the precentral cortex of patients with ALS, due to the higher sensitivity of multiecho $\mathrm{T} 2^{\star}$-weighted imaging to iron in the form of ferritin ${ }^{23}$; hence, their use was preferred in this study.

The sequence used in this study has often been used in the assessment of brain iron deposits ${ }^{24,25}$; however, it has recently been demonstrated that techniques that rely on the signal phase, namely quantitative susceptibility mapping, are more accurate than transverse relaxation times in terms of iron quantification. ${ }^{26,27}$ Nevertheless, the production of susceptibility maps requires particular acquisition settings: in most quantitative susceptibility mapping implementations such as ours, the MR imaging system is programmed to provide the complex MR imaging data divided into real and imaginary parts, which are of no radiologic use. In fact, quantitative susceptibility mapping requires additional scan time in addition to the conventional $3 \mathrm{D} \mathrm{T} 2^{\star}$ multiecho sequence that is included in the clinical protocol. Quantitative susceptibility mapping also requires time-consuming postprocessing.

A limitation of this study was that patients with ALS investigated at 3T and 7T were not the same; hence, a direct comparison of the diagnostic accuracies obtained with the systems working at different magnetic field strengths would be unfair. However, on the basis of the absence of significant differences in total and mean UMN scores between the 2 groups of patients and on the significant correlation of signal intensity and thickness with UMN scores, ${ }^{13}$ we could hypothesize that MR imaging morphologic changes of M1 are comparable between groups, and a cautious comparison of MR diagnostic accuracy between different magnetic fields could be made. A further limitation is the number of subjects enrolled. Considering that ALS is a rare disease, the population we investigated is quite large, but the potential clinical applications described above need to be tested on a larger group of subjects or, at least, on a different cohort of patients to confirm the feasibility and reproducibility of results.

\section{CONCLUSIONS}

The SH/Thk of the deep layers of the M1 measured with a semiautomated method at $3 \mathrm{~T}$ seems to be a radiologic marker of upper motor neuron burden in patients with ALS, though with less accuracy than at 7T.

Despite the heterogeneous magnitude of the UMN burden of patients, the combination of visual imaging assessment and the use of a semiautomated algorithm able to assess both thickness and $\mathrm{T}^{*}$ hypointensity of the deep layers of M1 could increase the sensitivity in evaluating images of patients referred with suspected motor neuron disease.

Disclosures: Mirco Cosottini-RELATED: Grant: Italian Ministry of Health, Com ments: This study has received funding (RF-2009-1546281) to Mirco Cosottini as Principal Investigator by the Italian Ministry of Health (www.salute.gov.it) and the Health Service of Tuscany (www.regione.toscana.it/sst). The funders had no role in study design, data collection and analysis, decision to publish, or preparation of the manuscript.* *Money paid to the institution.

\section{REFERENCES}

1. Brooks BR, Miller RG, Swash M, et al; World Federation of Neurology Research Group on Motor Neuron Diseases. El Escorial revisited: revised criteria for the diagnosis of amyotrophic lateral sclerosis. Amyotroph Lateral Scler Other Motor Neuron Disord 2000;1:293-99 CrossRef Medline

2. Ravits JM, La Spada AR. ALS motor phenotype heterogeneity, focality, and spread: deconstructing motor neuron degeneration. $\mathrm{Neu}$ rology 2009;73:805-11 CrossRef Medline

3. Kiernan MC, Vucic S, Cheah BC, et al. Amyotrophic lateral sclerosis. Lancet 2011;377:942-55 CrossRef Medline

4. Eisen A, Swash M. Clinical neurophysiology of ALS. Clin Neurophysiol 2001;112:2190-201 CrossRef Medline

5. de Carvalho M, Dengler R, Eisen A, et al. Electrodiagnostic criteria for diagnosis of ALS. Clin Neurophysiol 2008;119:497-503 CrossRef Medline

6. Kawamata T, Akiyama $\mathrm{H}$, Yamada $\mathrm{T}$, et al. Immunologic reactions in amyotrophic lateral sclerosis brain and spinal cord tissue. Am J Pathol 1992;140:691-707 Medline

7. Kwan JY, Jeong SY, Van Gelderen P, et al. Iron accumulation in deep cortical layers accounts for MRI signal abnormalities in ALS: correlating 7 Tesla MRI and pathology. PLoS One 2012;7:e35241 CrossRef Medline

8. Verstraete E, Veldink JH, Hendrikse J, et al. Structural MRI reveals cortical thinning in amyotrophic lateral sclerosis. J Neurol Neurosurg Psychiatry 2012;83:383-88 CrossRef Medline

9. Schuster C, Kasper E, Machts J, et al. Focal thinning of the motor cortex mirrors clinical features of amyotrophic lateral sclerosis and 
their phenotypes: a neuroimaging study. J Neurol 2013;260: 2856-64 CrossRef Medline

10. Walhout R, Westeneng HJ, Verstraete E, et al. Cortical thickness in ALS: towards a marker for upper motor neuron involvement. J Neurol Neurosurg Psychiatry 2015;86:288-94 CrossRef Medline

11. Foerster BR, Welsh RC, Feldman EL. 25 years of neuroimaging in amyotrophic lateral sclerosis. Nat Rev Neurol 2013;9:513-24 CrossRef Medline

12. Filippi M, Agosta F, Abrahams S, et al; European Federation of Neurological Societies. EFNS guidelines on the use of neuroimaging in the management of motor neuron diseases. Eur J Neurol 2010;17: 526-e20 CrossRef Medline

13. Cosottini M, Donatelli G, Costagli M, et al. High-resolution 7T MR imaging of the motor cortex in amyotrophic lateral sclerosis. $A J N R$ Am J Neuroradiol 2016;37:455-61 CrossRef Medline

14. Yousry TA, Schmid UD, Alkadhi H, et al. Localization of the motor hand area to a knob on the precentral gyrus: a new landmark. Brain 1997;120:141-57 CrossRef Medline

15. Penfield W, Rasmussen T. The Cerebral Cortex of Man: A Clinical Study of Localization of Function. New York: Macmillan; 1950

16. Costagli M, Donatelli G, Biagi L, et al. Magnetic susceptibility in the deep layers of the primary motor cortex in amyotrophic lateral sclerosis. Neuroimage Clin 2016;12:965-69 CrossRef Medline

17. Mochizuki Y, Mizutani T, Takasu T. Amyotrophic lateral sclerosis with marked neurological asymmetry: clinicopathological study. Acta Neuropathol 1995;90:44-50 CrossRef Medline

18. Kassubek J, Unrath A, Huppertz HJ, et al. Global brain atrophy and corticospinal tract alterations in ALS, as investigated by voxelbased morphometry of 3-D MRI. Amyotroph Lateral Scler Other Motor Neuron Disord 2005;6:213-20 CrossRef Medline
19. Devine MS, Pannek K, Coulthard A, et al. Exposing asymmetric gray matter vulnerability in amyotrophic lateral sclerosis. Neuroimage Clin 2015;7:782-87 CrossRef Medline

20. Hallgren B, Sourander P. The effect of age on the non-haemin iron in the human brain. J Neurochem 1958;3:41-51 CrossRef Medline

21. Bede P, Bokde A, Elamin M, et al. Grey matter correlates of clinical variables in amyotrophic lateral sclerosis (ALS): a neuroimaging study of ALS motor phenotype heterogeneity and cortical focality. J Neurol Neurosurg Psychiatry 2013;84:766-73 CrossRef Medline

22. Ignjatović $A$, Stević $Z$, Lavrnić $S$, et al. Brain iron MRI: a biomarker for amyotrophic lateral sclerosis. J Magn Reson Imaging 2013;38: 1472-79 CrossRef Medline

23. Adachi Y, Sato N, Saito Y, et al. Usefulness of SWI for the detection of iron in the motor cortex in amyotrophic lateral sclerosis. J Neuroimaging 2015;25:443-51 CrossRef Medline

24. Du G, Lewis MM, Styner M, et al. Combined R2 ${ }^{\star}$ and diffusion tensor imaging changes in the substantia nigra in Parkinson's disease. Mov Disord 2011;26:1627-32 CrossRef Medline

25. Baudrexel S, Nürnberger L, Rüb U, et al. Quantitative mapping of T1 and $\mathrm{T} 2^{\star}$ discloses nigral and brainstem pathology in early Parkinson's disease. Neuroimage 2010;51:512-20 CrossRef Medline

26. Schweser F, Deistung A, Lehr BW, et al. Quantitative imaging of intrinsic magnetic tissue properties using MRI signal phase: an approach to in vivo brain iron metabolism? Neuroimage 2011;54: 2789-807 CrossRef Medline

27. Barbosa JH, Santos AC, Tumas V, et al. Quantifying brain iron deposition in patients with Parkinson's disease using quantitative susceptibility mapping, R2 and R2. Magn Reson Imaging 2015;33: 559-65 CrossRef Medline 